\title{
LOS PLANES DE DESARROLLO DE LOS GOBIERNOS LOCALES DEL VALLE DEL MANTARO DESDE LA ÓPTICA DE LA SOSTENIBILIDAD, AÑO 2009
}

\author{
Hugo Miguel Miguel ${ }^{1}$, Jesús Hurtado Rivera ${ }^{2}$ \\ Facultad de Ciencias Forestales y del Ambiente de la Universidad Nacional del Centro del Perú
}

\begin{abstract}
RESUMEN
La investigación tuvo como objetivo determinar la orientación de los planes de desarrollo de los gobiernos locales del Valle del Mantaro en función de los principios del desarrollo sostenible; asimismo, analizar la estructura de los planes de desarrollo de los gobiernos locales en las dimensiones: ambiental, social, económico y político institucional. Para el análisis, se utilizaron los planes aprobados y publicados de cuatro municipios del Valle del Mantaro. Se concluye que los planes de desarrollo de los gobiernos locales del Valle del Mantaro no están orientados hacia el desarrollo sostenible. En la visión, no incluyen el paradigma de la sostenibilidad; finalmente, no obstante que incluyen los ejes y objetivos estratégicos de desarrollo social, económico, ambiental e institucional, el enfoque es lineal y no el sistémico.
\end{abstract}

Palabras clave: Gobiernos locales, sostenibilidad.

\section{DEVELOPMENT PLANS OF LOCAL GOVERNMENTS FROM Mantaro Valley perspective of sustainability, YEAR} 2009

\begin{abstract}
The study aimed to determine the orientation of the development plans of local governments in the Mantaro Valley in terms of the principles of sustainable development; also analyze, the structure of the development plans of local governments in the Environmental, social, economic and politic institutional dimensions. For the analysis were used the plans approved and published in four municipalities of the Mantaro Valley. We conclude that the development plans of local governments in the Mantaro Valley are not oriented towards sustainable development. In the vision, do not include the sustainability paradigm, and finally, however, including the axles and strategic objectives of social, economic, environmental and institutional, the approach is linear and not systemic.
\end{abstract}

Key words: Local governments, sustainability.

\footnotetext{
1 Ingeniero Forestal. Facultad de Ciencias Forestales y del Ambiente. E-mail: hmiguel21@gmail.com

2 Ingeniero Forestal. Facultad de Ciencias Forestales y del Ambiente. E-mail: jahr38@hotmail.com
} 


\section{INTRODUCCIÓN}

La función fundamental de los gobiernos locales, como parte de la estructura del estado e instancia de gobierno más próxima a la ciudadanía, es la elaboración y desarrollo de políticas públicas que contribuyan a asegurar el bienestar integral de sus poblaciones, posibiliten la construcción de ciudadanías responsables y garanticen el derecho de hombres y mujeres a elegir un lugar en el mundo donde puedan realizar sus expectativas y proyectos de vida, (2 y 7 ).

Por lo tanto, es muy importante la elaboración de los instrumentos de gestión de desarrollo de los gobiernos locales, dentro de estos, los planes de desarrollo concertado, que deben ser direccionados hacia el desarrollo sostenible, entendiendo este paradigma como un modelo de desarrollo equilibrado e interrelacionado entre las dimensiones económicas, sociales y ambientales de la localidad (7).

La investigación tuvo como objetivo determinar si los planes de desarrollo de los gobiernos locales del Valle del Mantaro, están orientados bajo los principios del desarrollo sostenible; asimismo, analizar la estructura de estos planes en las dimensiones: ambiental, social, económico y político institucional.

\section{MATERIALES Y MÉTODOS}

Para el análisis de los planes de desarrollo de los gobiernos locales del Valle del Mantaro, se utilizó una muestra no probabilística intencionada, por las facilidades y disponibilidad digital del instrumento de gestión de desarrollo; definiéndose cuatro gobiernos locales: Municipalidad Provincial de Huancayo, Municipalidad Provincial de Concepción, Municipalidad Provincial de Jauja, y Municipalidad Distrital de Huayucachi.

En función de la matriz de operacionalización de la variable principal de la investigación (plan de desarrollo concertado de gobiernos locales), se diseñaron fichas de evaluación de sostenibilidad; con la finalidad de evaluar los niveles de sostenibilidad de los planes de desarrollo concertado de las municipalidades estudiadas.

\section{Operacionalización de las variables:}

\begin{tabular}{|c|c|c|c|c|}
\hline $\begin{array}{c}\text { Variabl } \\
\mathrm{e}\end{array}$ & Indicadores & Índices & $\begin{array}{c}\text { Instrumen } \\
\text { tos de } \\
\text { medición }\end{array}$ & Fuente \\
\hline \multirow{2}{*}{$\begin{array}{l}\text { Plan de } \\
\text { desarro } \\
\text { llo } \\
\text { concert } \\
\text { ado de } \\
\text { gobiern }\end{array}$} & \multirow[b]{2}{*}{ Diagnóstico } & $\begin{array}{l}\text { Potencialid } \\
\text { ades }\end{array}$ & $\begin{array}{l}\text { Fichas de } \\
\text { evaluación }\end{array}$ & $\begin{array}{l}\text { Documento } \\
\text { digital }\end{array}$ \\
\hline & & $\begin{array}{l}\text { Elementos } \\
\text { históricos } \\
\text { culturales }\end{array}$ & $\begin{array}{l}\text { Fichas de } \\
\text { evaluación }\end{array}$ & $\begin{array}{l}\text { Documento } \\
\text { digital }\end{array}$ \\
\hline
\end{tabular}

\begin{tabular}{|c|c|c|c|c|}
\hline \multirow[t]{4}{*}{$\begin{array}{l}\text { os } \\
\text { locales }\end{array}$} & \multirow{3}{*}{$\begin{array}{l}\text { Ejes y } \\
\text { objetivos } \\
\text { estratégicos }\end{array}$} & $\begin{array}{l}\text { Visión de } \\
\text { desarrollo } \\
\text { sostenible } \\
\text { Eje de } \\
\text { desarrollo } \\
\text { social } \\
\text { Eje de } \\
\text { desarrollo } \\
\text { económico }\end{array}$ & $\begin{array}{l}\text { Fichas de } \\
\text { evaluación }\end{array}$ & $\begin{array}{l}\text { Documento } \\
\text { digital }\end{array}$ \\
\hline & & $\begin{array}{l}\text { Eje de } \\
\text { desarrollo } \\
\text { ambiental }\end{array}$ & $\begin{array}{l}\text { Fichas de } \\
\text { evaluación }\end{array}$ & $\begin{array}{l}\text { Documento } \\
\text { digital }\end{array}$ \\
\hline & & $\begin{array}{l}\text { Eje de } \\
\text { desarrollo } \\
\text { instituciona } \\
\text { I }\end{array}$ & $\begin{array}{l}\text { Fichas de } \\
\text { evaluación }\end{array}$ & $\begin{array}{l}\text { Documento } \\
\text { digital }\end{array}$ \\
\hline & $\begin{array}{l}\text { Programas de } \\
\text { inversión } \\
\text { multianual }\end{array}$ & $\begin{array}{l}\text { Sostenibili } \\
\text { dad }\end{array}$ & $\begin{array}{l}\text { Fichas de } \\
\text { evaluación }\end{array}$ & $\begin{array}{l}\text { Documento } \\
\text { digital }\end{array}$ \\
\hline
\end{tabular}

\section{RESULTADOS}

\subsection{Denominación de los planes de desarrollo locales:}

Tabla 1. Denominación de los planes de desarrollo locales.

\begin{tabular}{ll}
\hline \multicolumn{1}{c}{ Gobiernos Locales } & Denominación del plan de desarrollo: \\
\hline 1. Municipalidad & "Plan de desarrollo urbano de Huancayo \\
Provincial de Huancayo & 2006-2011" (PDU). \\
2. Municipalidad & "Plan de desarrollo provincial concertado \\
Provincial de Concepción & 2003-2015" \\
3. Municipalidad & "Plan de desarrollo urbano de la provincia \\
Provincial de Jauja & de Jauja. 2007-2010" \\
4. Municipalidad Distrital & Plan de desarrollo concertado 2009 - \\
de Huayucachi & 2020. \\
\hline
\end{tabular}

\subsection{Diagnóstico de las potencialidades tangibles e intangibles}

Tabla 2. Diagnóstico de las potencialidades tangibles e intangibles de los planes de desarrollo concertado de las municipalidades estudiadas.

\begin{tabular}{lcccc}
\hline & \multicolumn{3}{c}{ Diagnóstico Potencialidad } \\
Municipalidad: & $\begin{array}{c}\text { Potencialidades } \\
\text { Tangibles }\end{array}$ & $\begin{array}{c}\text { Potencialidades } \\
\text { Intangibles }\end{array}$ \\
\cline { 2 - 5 } & $\begin{array}{c}\text { Sí } \\
\text { incluyen }\end{array}$ & $\begin{array}{c}\text { No } \\
\text { incluye } \\
\text { n }\end{array}$ & $\begin{array}{c}\text { Sí } \\
\text { incluyen }\end{array}$ & $\begin{array}{c}\text { No } \\
\text { incluyen }\end{array}$ \\
\hline $\begin{array}{l}\text { Huancayo } \\
\text { Jauja }\end{array}$ & $\mathrm{X}$ & & $\mathrm{X}$ & \\
$\begin{array}{l}\text { Concepción } \\
\text { Huayucachi }\end{array}$ & $\mathrm{X}$ & & $\mathrm{X}$ & \\
$\begin{array}{l}\text { Porcentaje } \\
\text { (\%): }\end{array}$ & $\mathrm{X}$ & & $\mathrm{X}$ & \\
\hline
\end{tabular}

3.3 Diagnóstico de los elementos históricos y culturales Tabla 3. Diagnóstico de los elementos históricos y culturales de los planes de desarrollo concertado de las municipalidades estudiadas. 


\begin{tabular}{|c|c|c|c|c|c|c|c|c|}
\hline \multirow[t]{2}{*}{ alidad: } & \multicolumn{2}{|c|}{$\begin{array}{l}\text { Domesticaci } \\
\text { ón milenaria } \\
\text { de plantas y } \\
\text { animales }\end{array}$} & \multicolumn{2}{|c|}{$\begin{array}{l}\text { Conocimiento } \\
\text { s ancestrales }\end{array}$} & \multicolumn{2}{|c|}{$\begin{array}{l}\text { Técnicas } \\
\text { agrícolas } \\
\text { ancestrales }\end{array}$} & \multicolumn{2}{|c|}{$\begin{array}{l}\text { Conocimiento } \\
\mathrm{s} \text { adquiridos } \\
\text { en forma no } \\
\text { escolarizada }\end{array}$} \\
\hline & Sí & No & Sí & No & Sí & No & Sí & No \\
\hline $\begin{array}{l}\text { Huanca } \\
\text { yo }\end{array}$ & & $X$ & & $X$ & & $X$ & & $X$ \\
\hline Jauja & & $X$ & $X$ & & & $x$ & & $X$ \\
\hline $\begin{array}{l}\text { Concep } \\
\text { ción }\end{array}$ & & $X$ & $x$ & & $X$ & & & $X$ \\
\hline $\begin{array}{l}\text { Huayuc } \\
\text { achi }\end{array}$ & & $X$ & & $X$ & $x$ & & & $X$ \\
\hline $\begin{array}{l}\text { Porcent } \\
\text { aje (\%): }\end{array}$ & 0 & 100 & 50 & 50 & $\begin{array}{l}5 \\
0\end{array}$ & 50 & 0 & 100 \\
\hline
\end{tabular}

\subsection{Evaluación del paradigma de la visión de desarrollo}

Tabla 4. Evaluación del paradigma de la visión de desarrollo, incluidos en los planes de desarrollo concertado de las municipalidades estudiadas.

\begin{tabular}{lcccc}
\hline \multirow{2}{*}{$\begin{array}{l}\text { Municipalid } \\
\text { ad: }\end{array}$} & \multicolumn{2}{c}{$\begin{array}{c}\text { Tipo de Visión de Desarrollo } \\
\text { Paradigma del } \\
\text { desarrollo } \\
\text { sostenible }\end{array}$} & $\begin{array}{c}\text { Paradigma de } \\
\text { desarrollo } \\
\text { indefinido }\end{array}$ \\
\cline { 2 - 5 } & Sí & No & Sí & No \\
\hline Huancayo & X & & & X \\
Jauja & & X & X & \\
Concepción & & X & X & \\
Huayucachi & & X & X & \\
Porcentaje & 25 & 75 & 75 & 25 \\
(\%): & & & & \\
\hline
\end{tabular}

\subsection{Evaluación de los ejes y objetivos estratégicos}

Tabla 5. Evaluación de los ejes y objetivos estratégicos referidos en los planes de desarrollo concertado de las municipalidades estudiadas.

\begin{tabular}{|c|c|c|c|c|c|c|c|c|}
\hline \multirow{3}{*}{$\begin{array}{l}\text { Municipalidad } \\
:\end{array}$} & \multicolumn{8}{|c|}{ Ejes y Objetivos estratégicos } \\
\hline & \multicolumn{2}{|c|}{$\begin{array}{c}\text { Eje de } \\
\text { desarr } \\
\text { ollo } \\
\text { social }\end{array}$} & \multicolumn{2}{|c|}{$\begin{array}{c}\text { Eje de } \\
\text { desarrollo } \\
\text { económico }\end{array}$} & \multicolumn{2}{|c|}{$\begin{array}{c}\text { Eje de } \\
\text { desarrollo } \\
\text { ambiental }\end{array}$} & \multicolumn{2}{|c|}{$\begin{array}{c}\text { Eje de } \\
\text { desarroll } \\
0 \\
\text { institucio } \\
\text { nal } \\
\end{array}$} \\
\hline & Sí & No & Sí & No & Sí & No & Sí & No \\
\hline Huancayo & $X$ & & $X$ & & $X$ & & & $X$ \\
\hline Jauja & $X$ & & $x$ & & $X$ & & & $X$ \\
\hline Concepción & $X$ & & $X$ & & $X$ & & & $X$ \\
\hline Huayucachi & $X$ & & $X$ & & $X$ & & $X$ & \\
\hline $\begin{array}{l}\text { Porcentaje } \\
(\%):\end{array}$ & 00 & 0 & 100 & 0 & 100 & 0 & 25 & 75 \\
\hline
\end{tabular}

\subsection{Evaluación de la sostenibilidad de los programas de inversión multianual}

Tabla 6. Evaluación del "sello" de la sostenibilidad de los programas de inversión multianual.

\begin{tabular}{lcc}
\hline & \multicolumn{2}{c}{ Programas de inversión multianual } \\
Municipalidad: & $\begin{array}{c}\text { Proyectos } \\
\text { multidimensionales }\end{array}$ & $\begin{array}{c}\text { Proyectos con } \\
\text { enfoques lineales }\end{array}$ \\
\hline
\end{tabular}

\begin{tabular}{lcccc}
\cline { 2 - 5 } & Sí & No & Sí & No \\
\hline Huancayo & & $X$ & $X$ & \\
Jauja & & $X$ & $X$ & \\
Concepción & & $X$ & $X$ & \\
Huayucachi & & $X$ & $X$ & \\
Porcentaje (\%): & 0 & 100 & 100 & 0 \\
\hline
\end{tabular}

\section{DISCUSIÓN}

Según el Artículo 20 de la Ley Orgánica de Municipalidades 27972, (16), el nombre correcto que se les asigna a los planes de desarrollo de los gobiernos locales, se denomina: "Plan Integral de Desarrollo Sostenible Local"; no obstante, según la Tabla 1, los municipios estudiados han denominado en forma distinta a sus planes de desarrollo; con lo que se demuestra la falta de dominio conceptual del paradigma de la sostenibilidad, (14).

La evaluación del diagnóstico de las potencialidades (tabla 2), de los planes de desarrollo de las municipalidades estudiadas, consideraron en la totalidad (100\%), las potencialidades tangibles, como las inversiones físicas, infraestructura de servicios, infraestructura para la producción y/o el transporte; los componentes de la naturaleza, suelos, ganado diverso, recursos mineros, etc.; y los recursos financieros.

Asimismo, en todos los casos, han incluido las potencialidades intangibles, que corresponden a distintos aspectos vinculados con la sociedad y/o con el medio geográfico, tales como los referidos a las condiciones medioambientales, como las plantas y animales propios de la zona; el paisaje natural, y las condiciones del suelo y del clima.

En relación con el diagnóstico de los elementos histórico culturales, en mayor porcentaje todos los planes de desarrollo no le han dado la importancia del caso, como se puede observar en la Tabla 3, lo que significa que no consideraron la importancia de la dimensión social de la sostenibilidad, aspecto que constituye vital para gestionar el desarrollo sostenible de cualquier localidad, (8 y 10). La direccionalidad de una gestión de un gobierno local se sintetiza en la visión de los planes de desarrollo; en efecto, los tres municipios (Jauja, Concepción y Huayucachi), en sus visiones no definen claramente los paradigmas relacionados con el desarrollo sostenible, contrariamente se puede percibir una confusión conceptual; por lo que se coincide con las apreciaciones hechas por Díaz (14), en la que al estudiar los planes de desarrollo local en América Latina, concluye que en esta parte del mundo tiene una realidad en el que los intentos para impulsar el modelo del desarrollo sostenible no han sido eficaces durante la pasada década; los pocos recursos, la falta de voluntad política, un acercamiento fragmentado y no coordinado y los continuos modelos derrochadores de producción y de consumo, han 
frustrado los esfuerzos de poner en ejecución el desarrollo sostenible, desarrollo humano y la aplicación de la Agenda 21 , entre las necesidades económicas y sociales de la gente y la capacidad de los recursos terrestres y de los ecosistemas para resolver necesidades presentes y futuras.

En todos los casos, los planes de desarrollo han incluido los tres ejes y objetivos estratégicos (social, económico y ambiental), obviando el eje de desarrollo institucional; sin embargo, los objetivos estratégicos y los programas de inversión multianual (tabla 6), han sido diseñados con una visión lineal; notándose claramente la falta del dominio conceptual del desarrollo sostenible, para generar objetivos estratégicos y proyectos específicos de tipo sistémico o multidimensionales que garanticen equilibradamente la sostenibilidad económica, la sostenibilidad social y la sostenibilidad ambiental, con la condición que estas estén totalmente relacionadas, (14).

\section{CONCLUSIONES}

- Los planes de desarrollo de los gobiernos locales del Valle del Mantaro, no están orientados bajo los principios del desarrollo sostenible.

- No obstante que los planes de desarrollo han incluido los tres ejes y objetivos estratégicos (social, económico y ambiental); los objetivos estratégicos y los programas de inversión multianual han sido diseñados con una visión lineal, alejados de los principios de la sostenibilidad.

\section{REFERENCIAS BIBLIOGRÁFICAS}

- Acción Local. El rol de los gobiernos locales latinoamericanos en el siglo 21. Santiago de Chile; 2003.

- Garzón LE. Los gobiernos locales en la esfera global. Bogotá D.C.; 2008

- $\quad$ Castillo P. El desarrollo local en la gestión municipal. Ciencias Sociales Online. Universidad de Viña del Mar. Chile; 2006.

- Montesinos G, Valdivia G. Roles y funciones del gobierno local. Red de municipalidades rurales del Perú. Lima; 2007.

- Vera A. Gobiernos locales: visión administrativa general. Escuela mayor de Gestión Municipal. Lima; 2006.

- $\quad$ García M. La Agenda 21 Local. Proceso y evaluación. Revista Galega de Economía, España. vol.13, núm. 12 (2004), pp 1-21.

- Mckeown R. Manual de educación para el desarrollo sostenible. Centro de Energía, Medio Ambiente y Recursos. Universidad de Tennessee, USA; 2002.
- $\quad$ Organización de las Naciones Unidas. Agenda 21. Programa de las Naciones Unidas para el Medio Ambiente; 1992.

- Crozier M, Gobiernos locales: liderazgo y gestión de los recursos humanos. Córdova, Argentina; 2001.

- Coria L. El rol de las autoridades locales para el desarrollo sostenible: la experiencia de los municipios de la microregión Anadagalá Pomán (Argentina). DELOS: Desarrollo Local Sostenible. Vol. 1, $\mathrm{N}^{\circ}$ 1; 2007.

- Morales M. El desarrollo local sostenible. Revista: Economía y Desarrollo- N 2 / Vol. 140/Jul.-Dic. / 2006.

- Vera A. Gobiernos locales: Visión administrativa general. Escuela Mayor de Gestión Municipal. Lima, Perú; 2002.

- Díaz J. Planes de gobierno regional y local. Propuestas marco de sugerencias para la incidencia sobre las organizaciones políticas que postulan a las elecciones regionales y municipales. Instituto para la Democracia Participativa. Lima, Perú; 2006.

- Díaz RL. Planes de desarrollo local: enfoques y tendencias en América Latina. Lima, Perú; 2003.

- $\quad$ Ley Orgánica de Municipalidades. Ley N 27972 